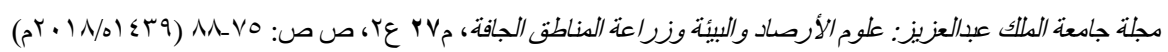
DOI: 10.4197/Met. 27-2.8

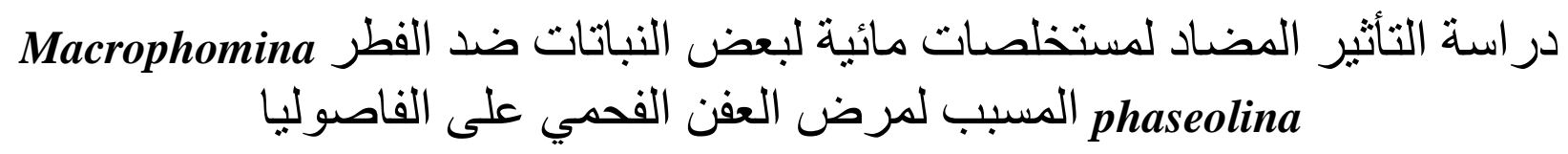

*أحمد علي الجمالي، وعبدالله نعمان الأيباني، وجمال عبده الشرعبي، ومروان عبده مانع

$$
\text { *قسم الإنتاج النباتي، كلية الزراعة بجامعة إب، الجمهورية البينية }
$$

المستخلص. تمت دراسة تأثير المستخلصات المائية لست نباتات من البيئة المحلية على نمو الفطر Macrophomina Phaseolina

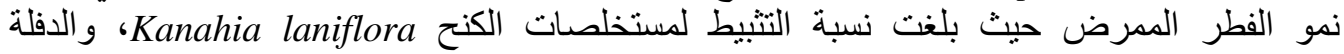

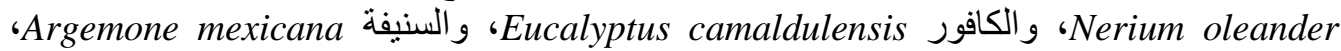

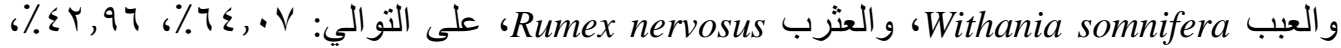
زيادة

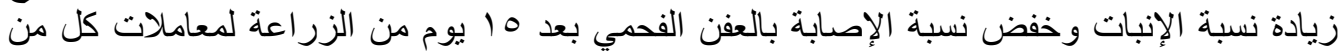

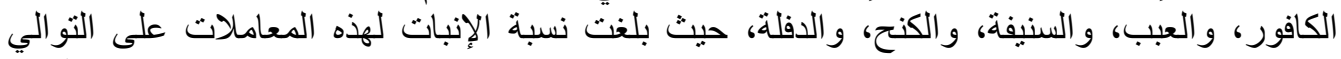

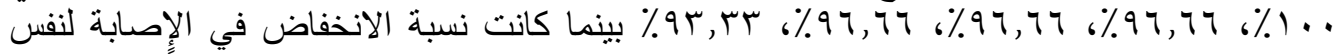

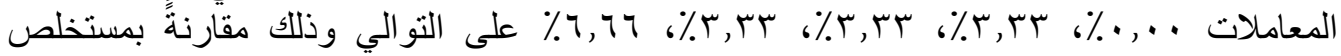

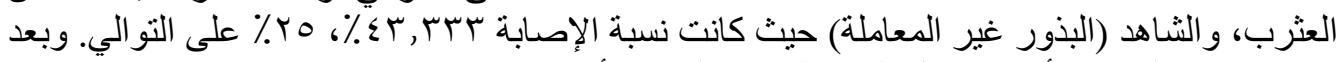

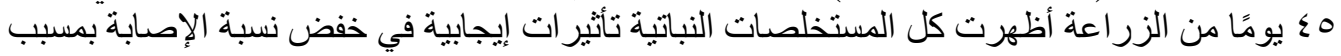

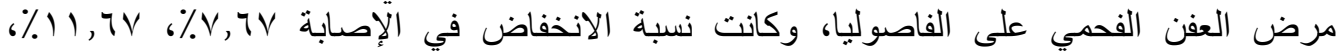

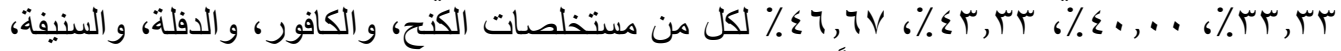

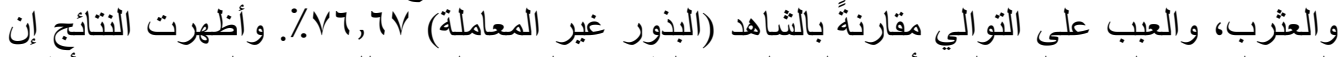

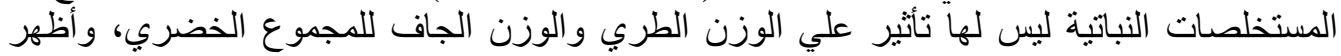

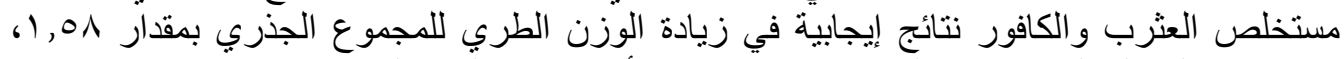

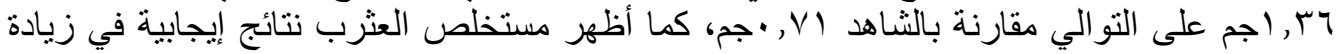

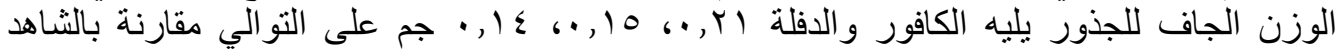

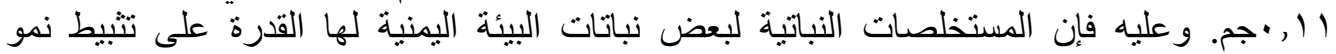

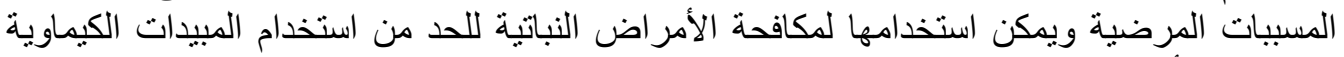

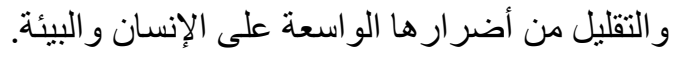

الكلمات الدالة: المستخلصات النباتية، أمر اض فطرية، التعفن الفحمي، الفاصوليا.

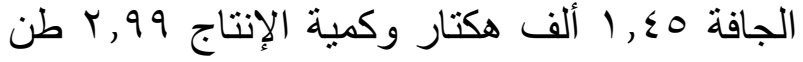

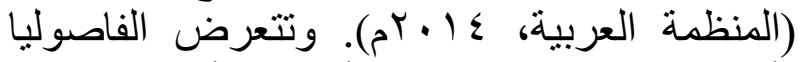

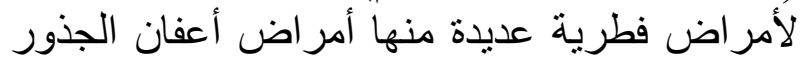

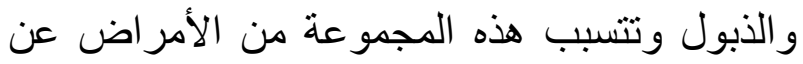

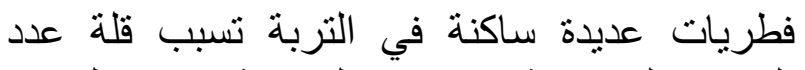
النباتات المنزرعة في وحدة المساحة وقد تصل فئل هذه

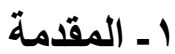

تعد الفاصوليا (vulgaris الاقتصادية والغذائية العالية، وتبلغ المساحة المزروعة في الجمهورية اليمنية من الفاصوليا 
وزيادة الاعتماد عليها يزيد من هذه التكلفة (لبابيدي و وزيشة، 990 (1) و و (Unger and Brideaux, 1997)

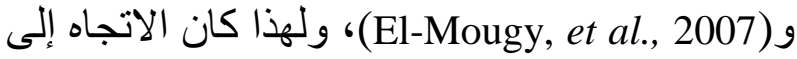
استخدام المبيدات الطبيعية والحصول على عركي مركبات

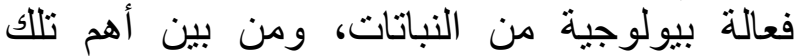
الطرق التي نالت اهتمامًا في العقدين الأخيرين هي

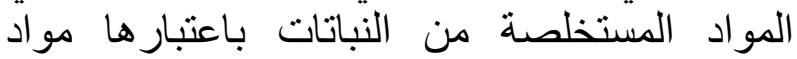

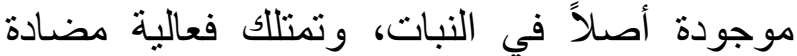

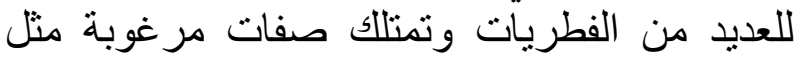

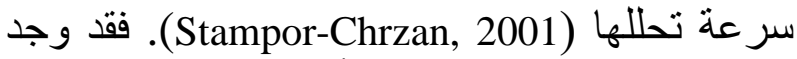
(الناصر و آخرون، ع ا • ب م) أن للمستخلص الزيتي

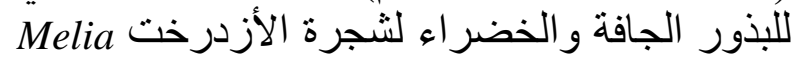
فعالية في تثبيط نمو الفطرين فotrytis cinerea Penicillium digitatum الوسط المغذي PDA في المخبر. أظهرت النتائج

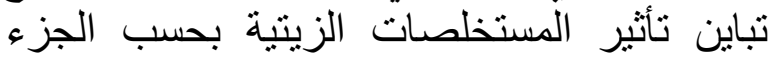
النباتي المستخدم. فقد تفوق مستخلص البذور الجافة

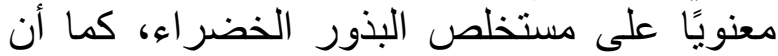

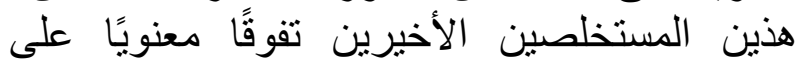
مستخلص الأز هار من حيث تأثنيره في نمو المشيجة، إذ توقف نمو الفطر B. cinerea تمامًا عند التركيز

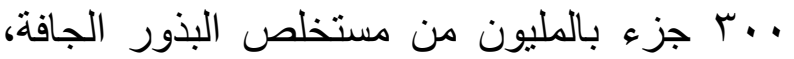

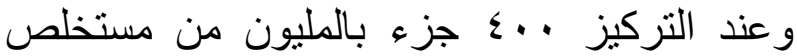

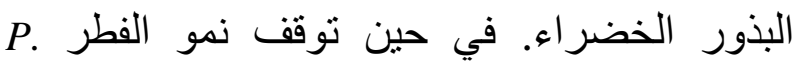
digitatum من مستخلص البذور الجافة، وكانت نسبة التثبيط

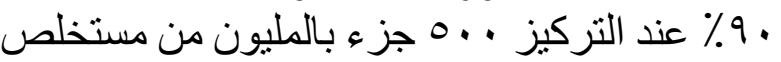
البذور الخضراء. ويهدف البحث إلى دهُ دراسة تأثير

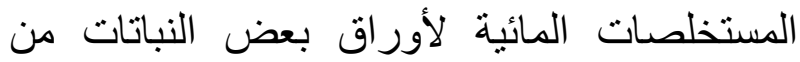

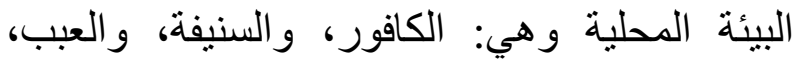
و العثرب، و الدفلة والكنح على مسبب مرض وض العن العفن Macrophomina الفحمي على باردات الفاصوليا phaseolina

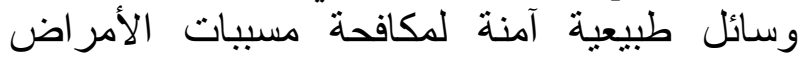
النباتية وخاصة على المحاصيل المهمة في بلادنا.

$$
\text { r ـ مواد وطرائق البحث }
$$

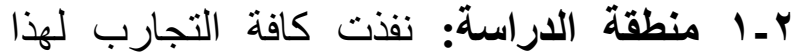
البحث في المختبر والصوبة الزجاجية لقسم الوقاية

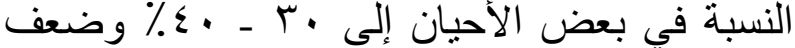

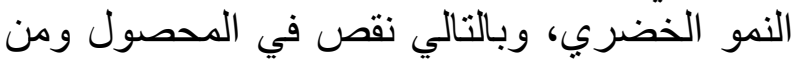

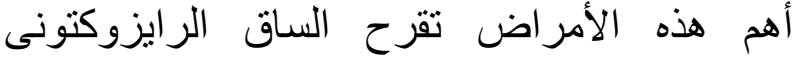

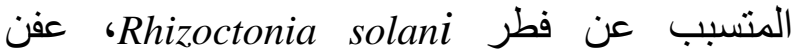
Fusarium الجذور الجاف المتسبب عن فطر

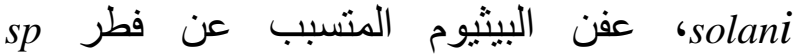
Pythium Sclerotium rolfsii

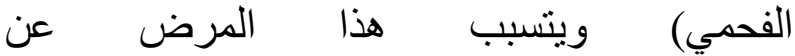
الفطر Macrophomina phaseolina ويعد هذا الفطر من الفطريات الناقصة التي تكون أجسامًا حجرية.

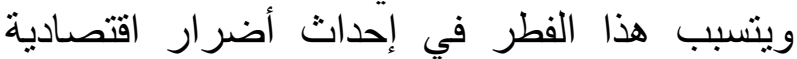
كبيرة لكثير من المحاصيل الزراعية بما فيها

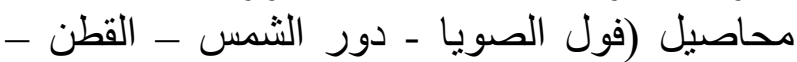

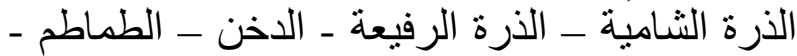

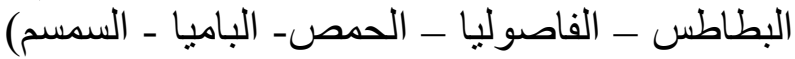

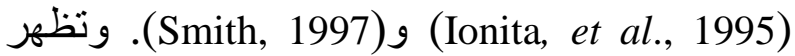
أعر اض الإصابة بالفطر M. phaseolina على نبات الفاصوليا بهيئة تقرح غائر أسود اللون ربما باصنا يتطور على قاعدة الساق وتؤدي الإصابة إلى موت التئ النبات

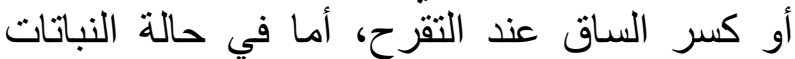

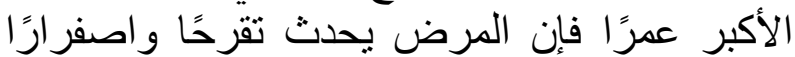

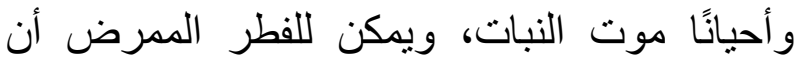
يكون كلا الطورين السكليروشي و والبكني في في لإني الأنسجة المصابة والتي تظهر بشكل بقع داكنة أو سوداء فحمية لذللك بسمى المرض الته عادة بالعفن

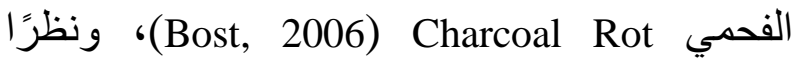
لاستخدام المبيدات الكيمائية المصنعة وبصورة عشوائية وغير مبرمجة للتقليل من مخاطر تلائلك

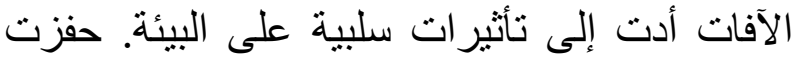

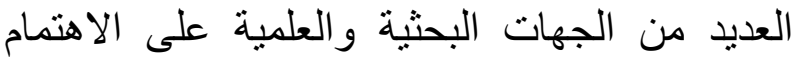

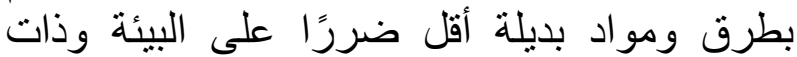
فاعلية جيدة في الحد من تأثير الآفات ( El-Morsi

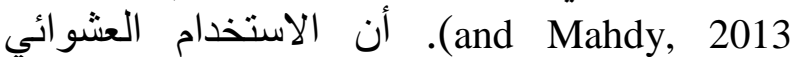
و اللاعقلاني لهذه المبيدات أدى إلى حصول تأثير ات

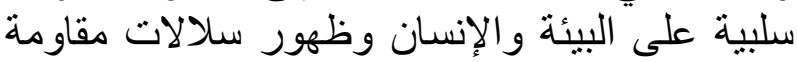
من الحشر ات ومسبيات الأمر اضل الئ، كما وأن المكافحة

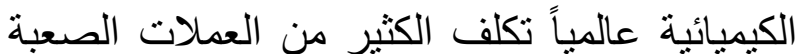


ع r ساعة ورجت بين كل فترة وأخرى وبعدها تم

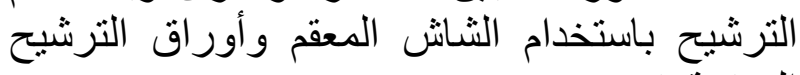
المعقمة (El-Fiki, et al., 2004).

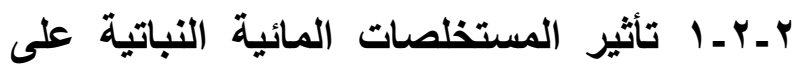
نمو الفطر Macrophomina phaseolina مختبريًا

:In-vitro

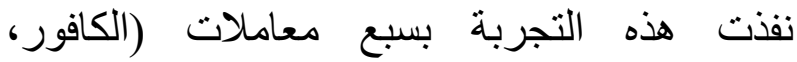
و النسيفة، و والعبب، و والعثرب، و والدفلة، و والكنح

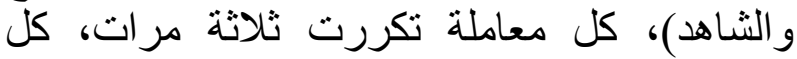
مكرر تم في طبق بتري قطره 9 سم. أضبفت المستخلصنات النباتية إلى البيئة الغذائية فئرة على حدة على حسب المعاملات وذللك قبل تجمد الكئل البيئة، وذلك بنسبة ا مل لكل 10 مل مل من البيئة

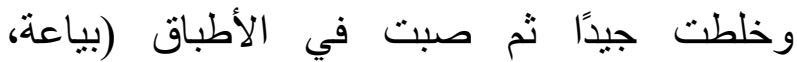

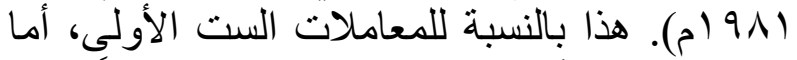

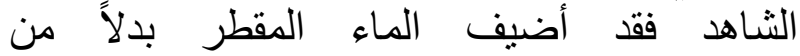
المستخلصات وبنفس النسبة وبعد أن تجمدت البيئة

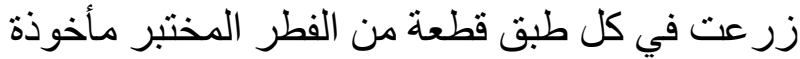

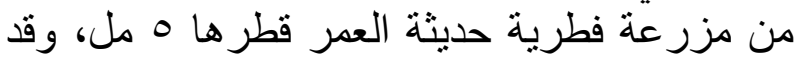

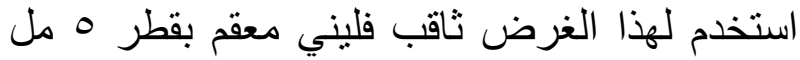

$$
\text { (الميسري، } 999 \text { (م). }
$$

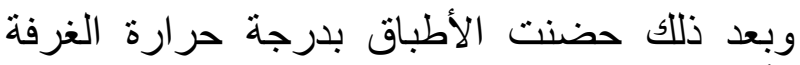

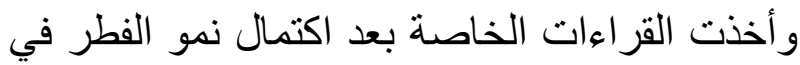
معاملة الثـاهد، وهي عبارة التهارة عن متوسط قطرين

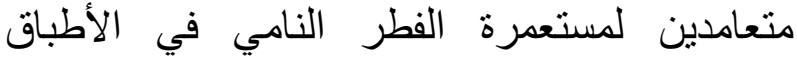

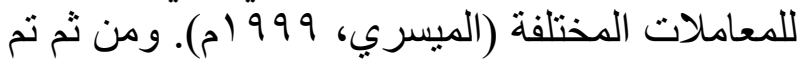
قياس نسبة التثبيط بحسب المعادلة الآتية (عبود

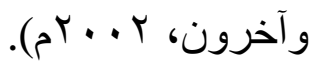

في كلية الزراعة والطب البيطري - جامعة إب،

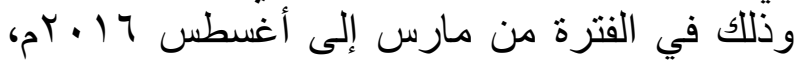

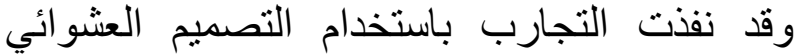
الكامل، وحللت البيانات إحصائيًا باستخدام جدول تحليل التباين وتم تقدير أقل فرق معنوي للمفاضلة

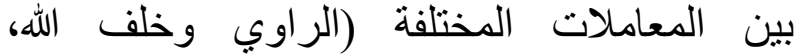

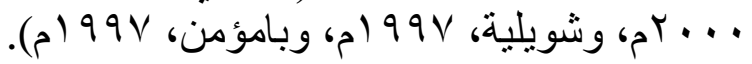

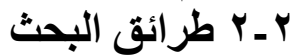

تحضير المستخلصات المائية النباتية: تم جمع الأوراق لكلٍ من نبات الكافور Eucalyptus sp،

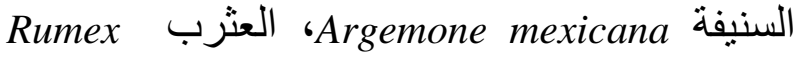
،Nerium oleander الدفنة ،nervosus Kanahia laniflora

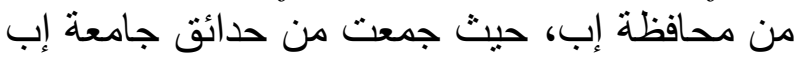

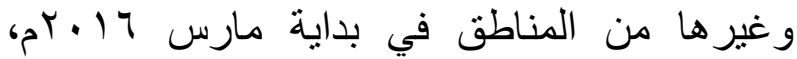

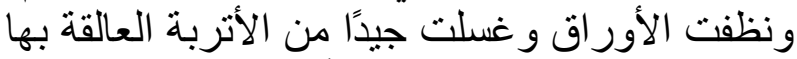

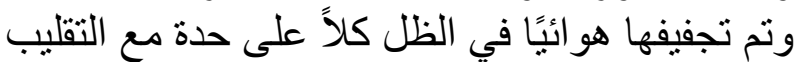

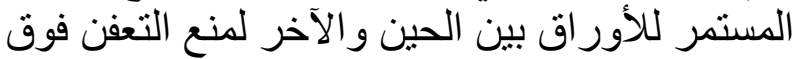

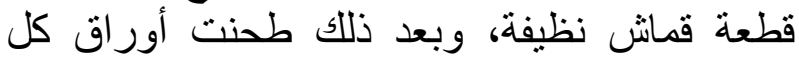
نبات على حدة بالخلاط الكهربائي وحفظت فلثة البودرة في أكياس بلاستيكية في الثلاجة لحة لحين الاستعمال

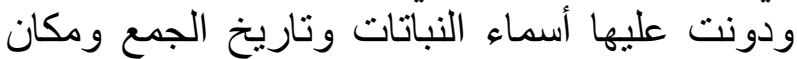
الجمع وذللك باعتماد طريقة (Riose, et al., 1987).

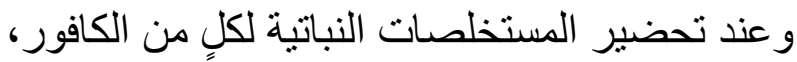

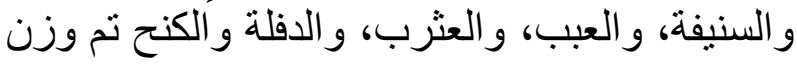

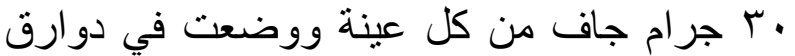

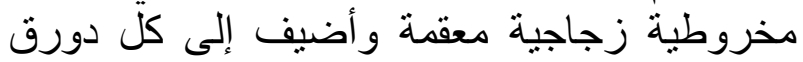

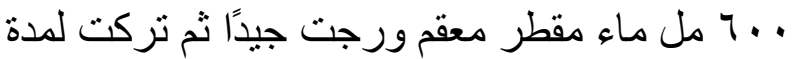

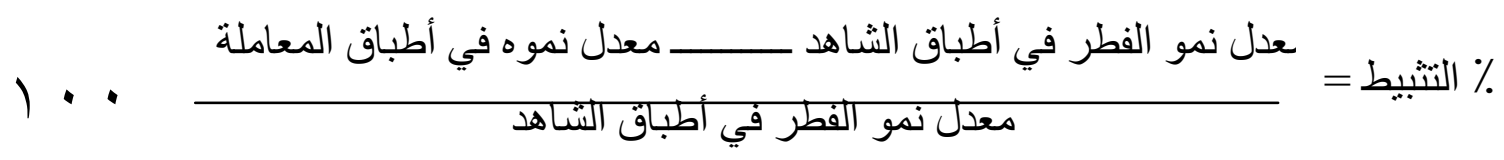

لمعرفة تأثثر معاملة البذور بالمستخلصات المائية النباتية على الإصابة بالفطر الممرض تحكية تحت سطح

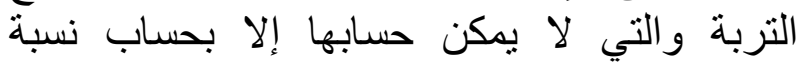

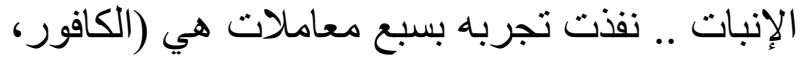

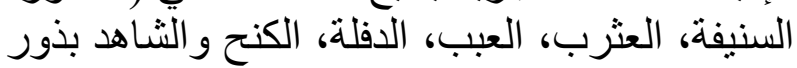

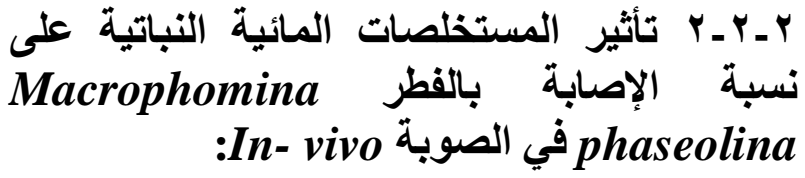




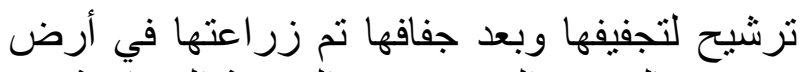

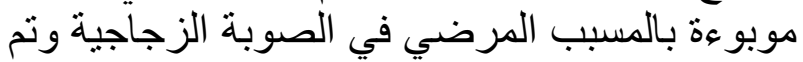

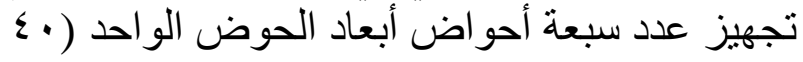

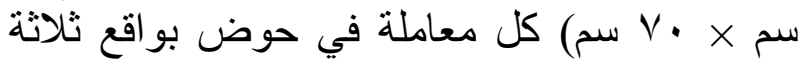

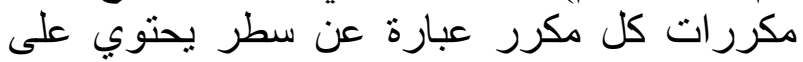

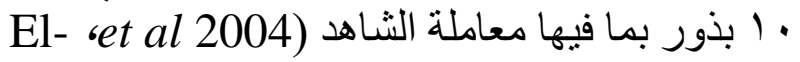

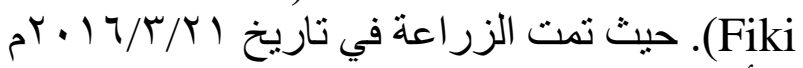

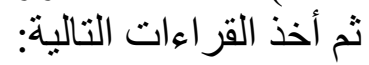

غير معاملة بالمستخلصات منقع في الماء المقطر

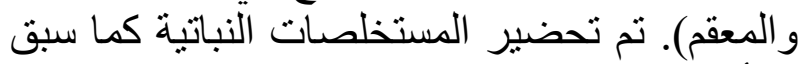

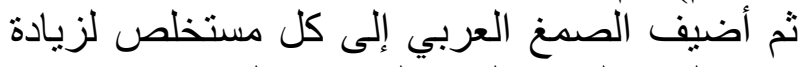

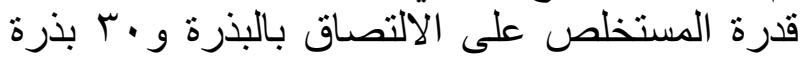

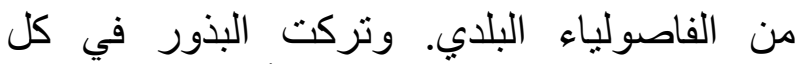

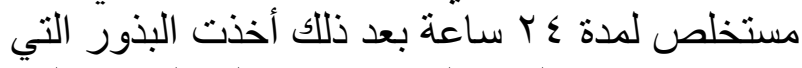

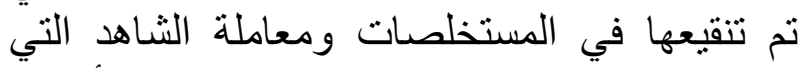
وضعت في ماء مقطر معقم ووضعت في أوراق

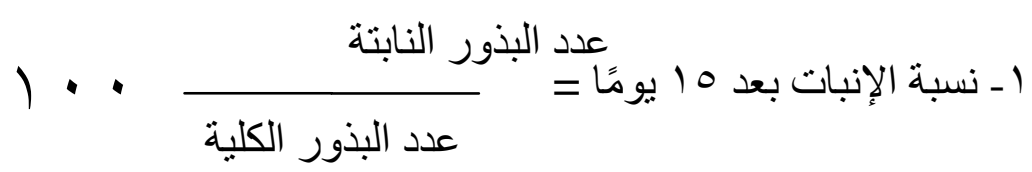

$$
\begin{aligned}
& \text { عدد البذور غير النابتة }
\end{aligned}
$$

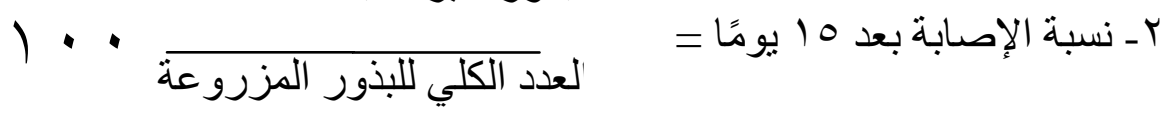

$$
\begin{aligned}
& \text { النباتات النابتة التي تظهر عليها الإصابة } \\
& \text { عدد النباتات النابتة الكلية } \\
& \text { " س- نسبة الإصابة بعد 0؛ يومًا }
\end{aligned}
$$

.(El-Mougy, et al., 2007)

نسبة تثبيط لنمو الفطر وبين معاملة كلاً من العثرب

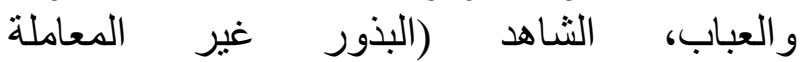
بالمستخلص)، ولا تظهر فروق معنوية بين الكافور، و الدفلة و السنيفة حيث كانت نسبة التتبيط لنمو الفطر

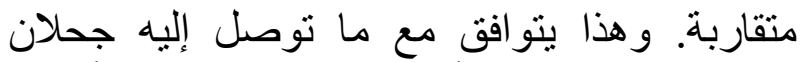

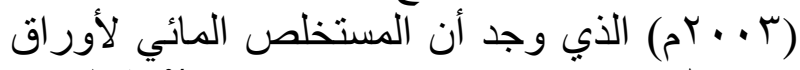

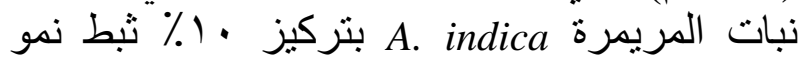
الفطر Macrophomina phaseolina في ظروف المختبر على البيئة الغذائية PDA ووصلت نسبة

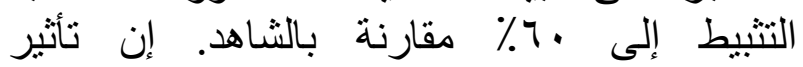

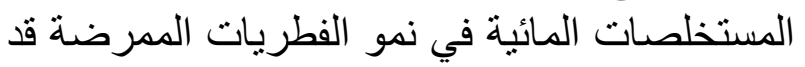

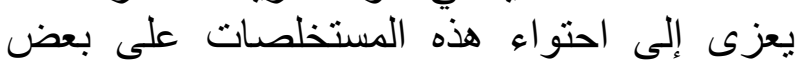

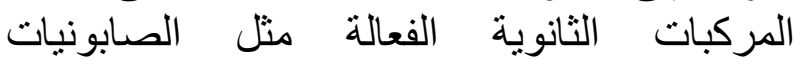
و الفينو لات لها القدرة على تثبيط نمو الفطريات (Papadopoulou, et al., 1999)

جدول (1). تأثير المستخلصات المائية النباتية على نمو الفطر Macrophomina Phaseolina في المختبرل)
عـ تحديد الوزن الطري و الجاف لكلاً من المجموع

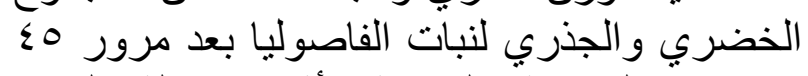

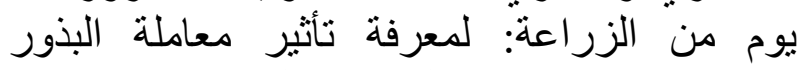
بالمستخلصات النباتية على النمو الخضري والكير والجذري لنبات الفاصوليا (EI-Fiki, et al., 2004). r. النتائج والمناقشة

r-1 أثأثير المستخلصات المائية النباتية على نمو الفطر Macrophomina Phaseolina في الثير المختبر :In vitro

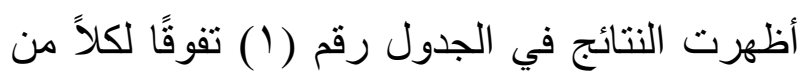

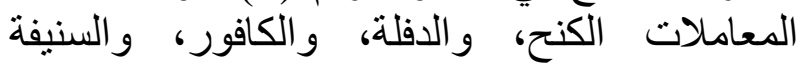

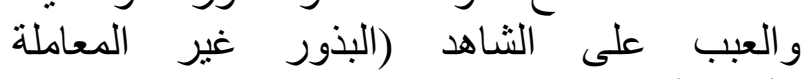

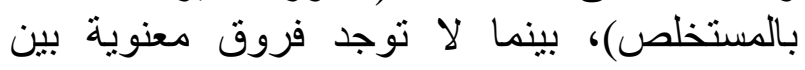

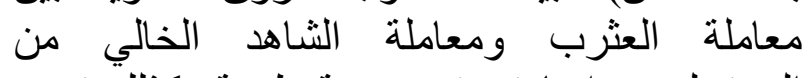

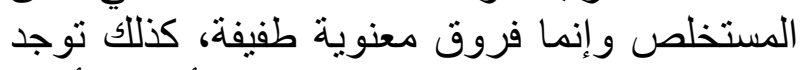

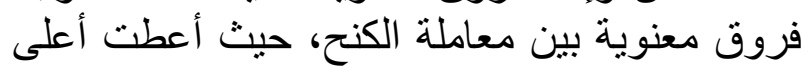


بعض العناصر الغذائية والأملاح الضرورية في

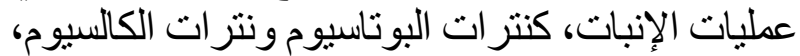

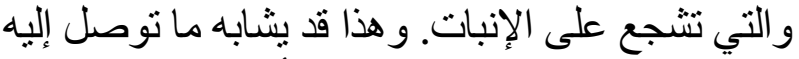
(Bhatt and Todaria, 1990) تختلف في مدى استجابتها لتأثير المستخلصات في نسبة

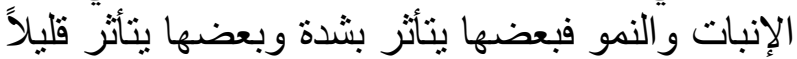
و البعض الآبات والنمر لا بتأثر.

كما أظهرت النتائج أنه لا توجد فروقًا معنوية بين

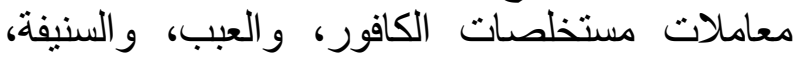

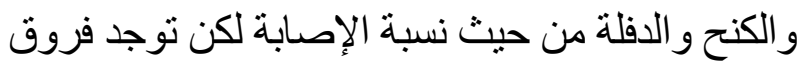

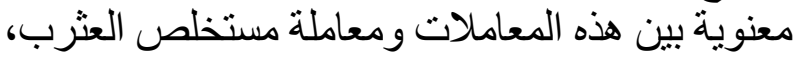

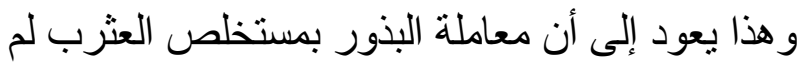

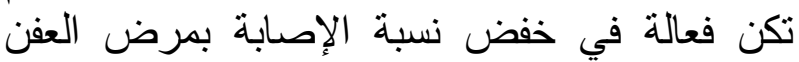

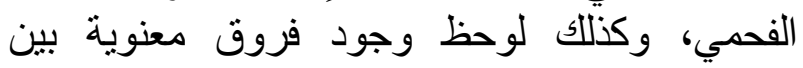

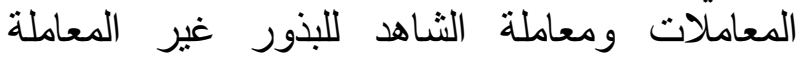

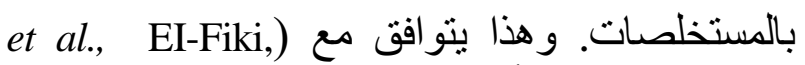

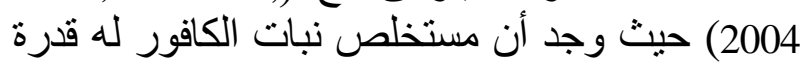
على خفض نسبة الإصابة بمرض العفن الفن الفحي على

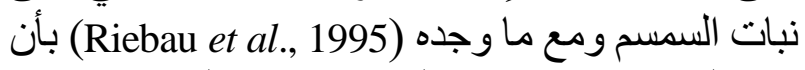
مستخلص زيوت نبات الكافور ومستخلصات زئات زيوت نباتات أخرى لها نشاط كمضاد فطري ضي ضد العديد من

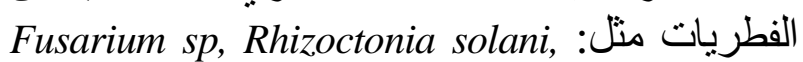
Macrophomina phaseolina.

جدول (r). تأثير المستخلصات النباتية على نسبة الإنبات

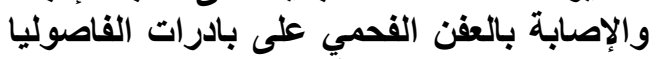

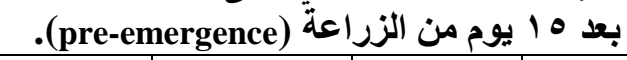

\begin{tabular}{|c|c|c|c|}
\hline $\begin{array}{c}\text { الإصابة الإضخفاض } \\
\text { reduction } \\
\text { [\%] }\end{array}$ & $\begin{array}{c}\text { \% المرضوث } \\
\text { incidence [\%] }\end{array}$ & \% للإنبات & $\begin{array}{c}\text { المعاملات } \\
\text { Treatment }\end{array}$ \\
\hline 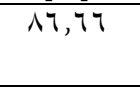 & $3.33 \mathrm{C}$ & $96.66 \mathrm{~A}$ & $\begin{array}{l}\text { Argemone السنيفة } \\
\text { mexicana L }\end{array}$ \\
\hline $1 \ldots$ & $0.00 \mathrm{C}$ & $100.00 \mathrm{~A}$ & $\begin{array}{c}\text { الكافور } \\
\text { Eucalyptu }\end{array}$ \\
\hline 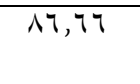 & $3.33 \mathrm{C}$ & $96.66 \mathrm{~A}$ & $\begin{array}{l}\text { Withania العبب } \\
\text { somnifera }\end{array}$ \\
\hline$V T$ & $43.33 \mathrm{~A}$ & $56.66 \mathrm{C}$ & $\begin{array}{l}\text { Rumex العثرب } \\
\text { nervosus Vahl }\end{array}$ \\
\hline VT,Tr & $6.66 \mathrm{C}$ & $93.33 \mathrm{~A}$ & $\begin{array}{c}\text { Nerium الدفلة } \\
\text { oleander }\end{array}$ \\
\hline$\wedge \neg, 7 \uparrow$ & $3.33 \mathrm{C}$ & $96.66 \mathrm{~A}$ & $\begin{array}{l}\text { Kanahia الكنح } \\
\text { laniflora }\end{array}$ \\
\hline- & $25.0 \mathrm{~B}$ & $75.0 \mathrm{~B}$ & الشاهد Control \\
\hline
\end{tabular}

\begin{tabular}{|c|c|}
\hline $\begin{array}{l}\text { Average growth \% } \\
\text { \% } \\
\text { reduction }\end{array}$ & $\begin{array}{c}\text { المعاملات } \\
\text { Treatment }\end{array}$ \\
\hline $42.58 \mathrm{AB}$ & Eucalyptus sp الكافور \\
\hline $33.48 \mathrm{~B}$ & العبب Withania somnifera \\
\hline $42.96 \mathrm{AB}$ & الدفلة Nerium oleander \\
\hline $64.07 \mathrm{~A}$ & الكنح Kanahia laniflora \\
\hline $22.96 \mathrm{BC}$ & Rumex nervosus Vahl العثرب \\
\hline $41.86 \mathrm{AB}$ & Argemone mexicana L السنيفة \\
\hline $0.00 \mathrm{C}$ & الشاهد Control \\
\hline \multicolumn{2}{|c|}{ 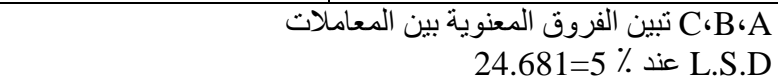 } \\
\hline
\end{tabular}

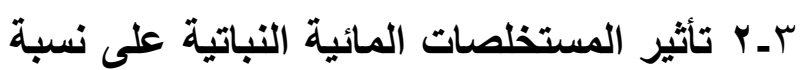

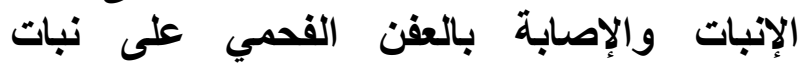
الفاصوليا في الصوبة الزجاجية الزبة الزبة

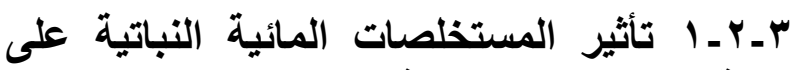

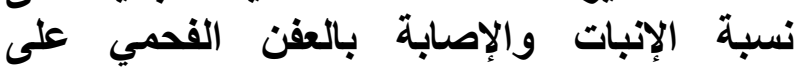
Pre - الفاصوليا بعد

\section{:emergence}

أظهرت النتائج في الجدول رقم (Y) عدم وجود أي فروق معنوية بين معاملات الكافور، و والسنيفة،

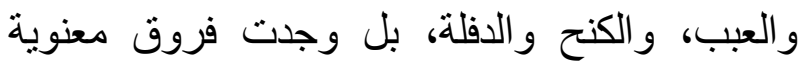
طفيفة نوضح نسبة الإنبات في هذه المعاملات، وبئه توجد هنالك فروقًاً معنوية بين الإنات فئه هذه المعاملات السابقة

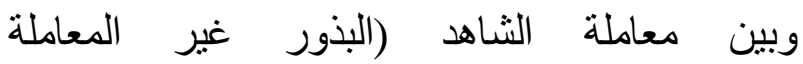

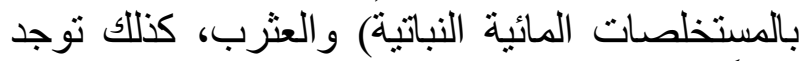

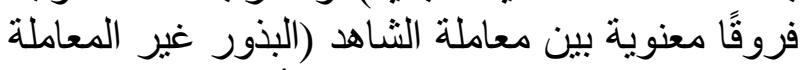

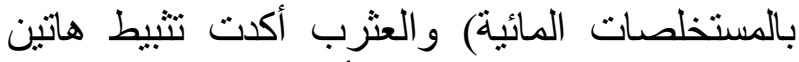

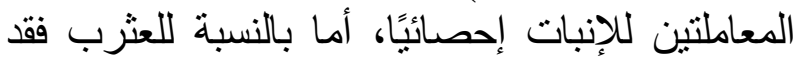

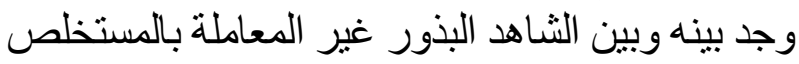

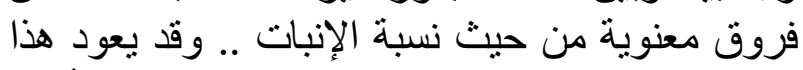

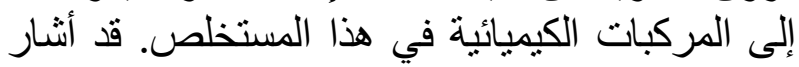

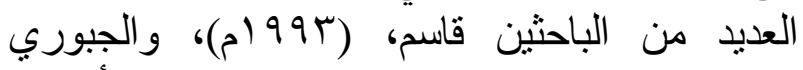

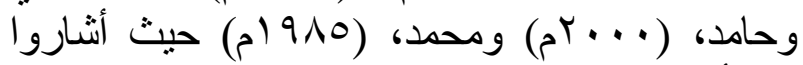
إلى أن النباتات المختلفة تختلف فيما بـان بينها في نسبة

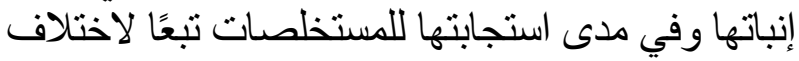
عواملها الَّراثية. هذا إضـافة إلى احتواء التهاء التربة على لإنى 


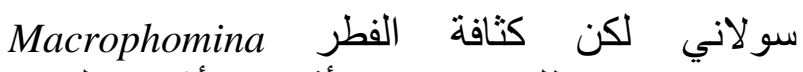
phaseolina

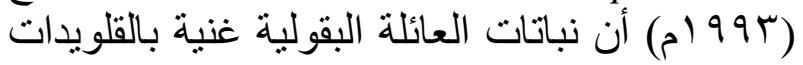

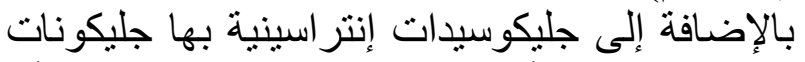

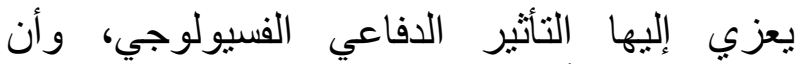

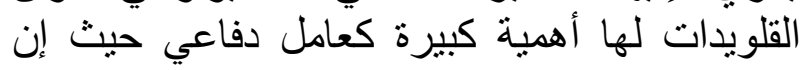

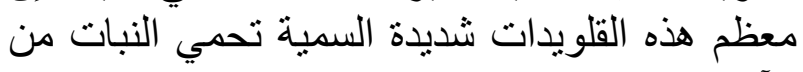

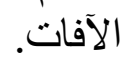

وقد أكد حجاج (Haggage, 1998) أن للقلويدات تأثثيرًا فعالاً ضدا ضد العديد من الفطريات ومنها .Fusarium solani

وكما وجد بران وآخرون (Paran, et al., 1996) أن مستخلص نبات الكافور. نبات الحلفابر نyات كاتت فعالة ولها نشاط الفاط مقاوم لإحدى عشر فطرًا من الفطريات الممرضة للنباتات، منها لإحثر الفطريات من الفطريات Macrophomina phaseolina 'F.oxysporum بالإضافة لفطريات أخرى، حيث التركيز الأدنى لهذه

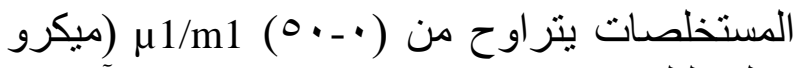

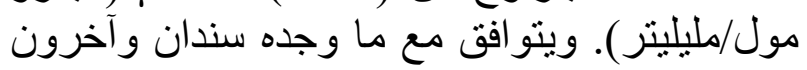
إن استخدام تركيزات (Sindhan, et al., 1999)

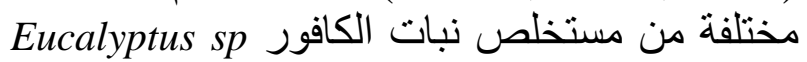

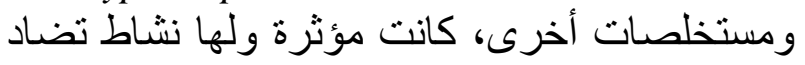

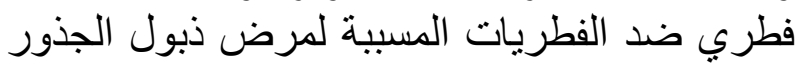

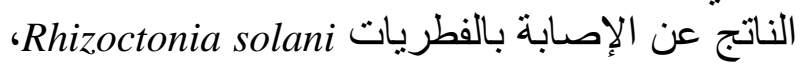
.Macrphomina phaseolina

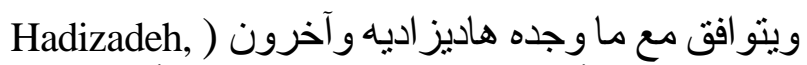
(et al., 2009

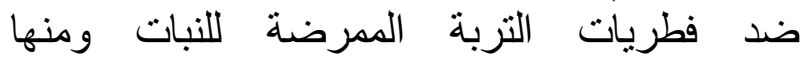
- Rhizoctonia solani ‘Alternaria altrnata . Fusarium oxysporum ‘Fusarium solani ويتوافق كذللك مع دراسة أجريت في الهند لتقييم فعالية عدد من مستخلصات النباتات التات الطبية ومنها نبات الدفلة ضد Alternaria solani حيث التئ أظهرت الدراسة تأثثر مثبط للمستخلصات النباتية ضد الفطر الثر

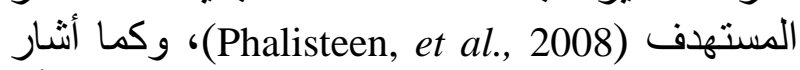
عثمان وآخرون (Osman, et al., 2007) أن (P) (P) \begin{tabular}{|l|l|l|l|}
\hline & 17.408 & 17.408 & 5\% ع L.S.D \\
\hline
\end{tabular}

نسبة الانخفاض في حدوث المرض reduction للمعاملات المختلفة مقارنة بمعاملة الثاهد البذاندة البذور

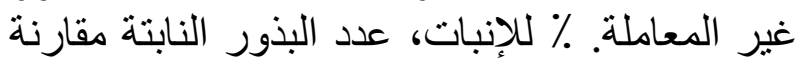

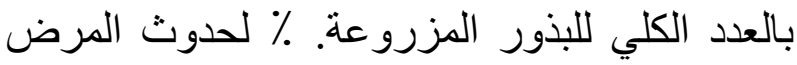

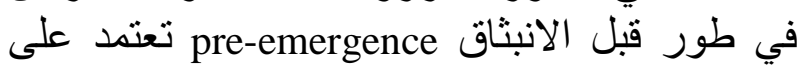
عدد البذور غير الانيتافة مقارنة بعدد البذور نعابذ

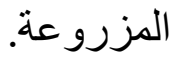

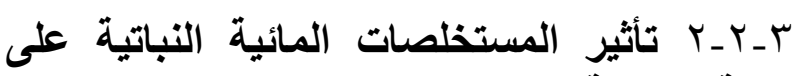

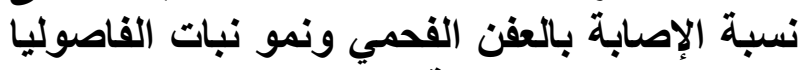

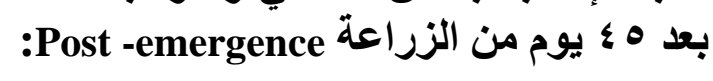

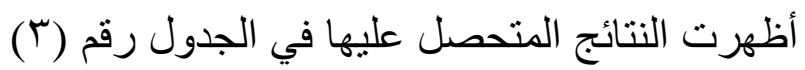

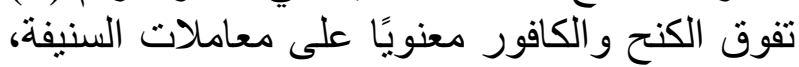

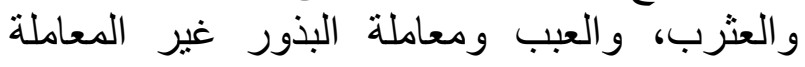

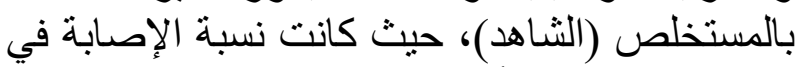

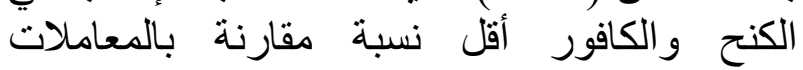

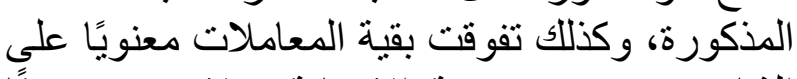

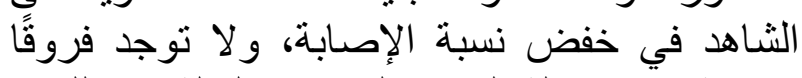

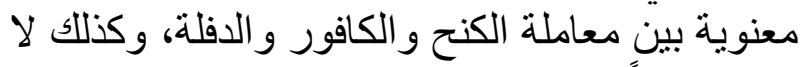

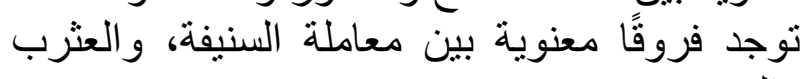
و العبب.

وقد يعود هذا التأثثر على الفطر المسبب للإصابة إلى كئى

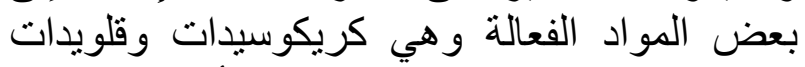

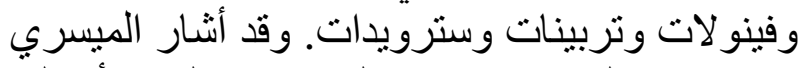

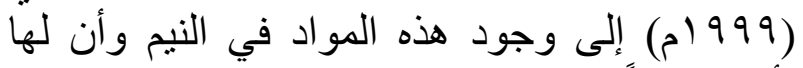

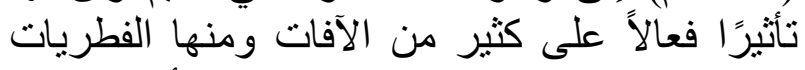

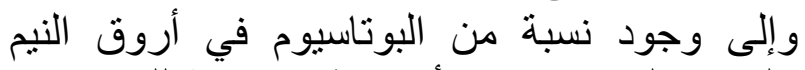

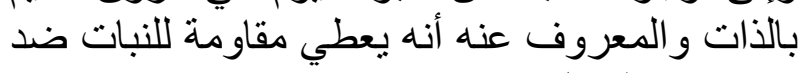

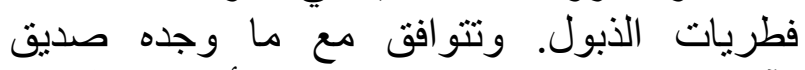
وآخرون (Siddiqui, et al., 2002) أن بقايا السنيفة المتحللة في التربة أدى إنى إلى انخفاض في كثافة

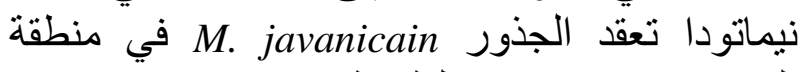

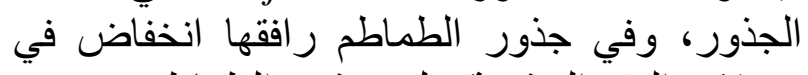
معدلات العقد الجذرية على جذور الطماطم رلف وحسنت من قياسات النمو للنبات.

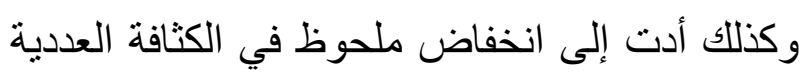
لكلا من فطري الفيوزاريوم سولاني ورايزوكتونيا لإنيا 
وحمض أكسالات البوتاسيوم Potassium oxalate

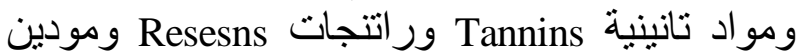
Emodin تحتوى جذوره مشتاقات الإنتر اكينون و الفلافونيدات، بينما تحتوي أوراقه على جليكوسيدات الإنتون الإنثراسين .Anthracene glycoside

وكذلك مستخلص نبات العبب Withania somnifera

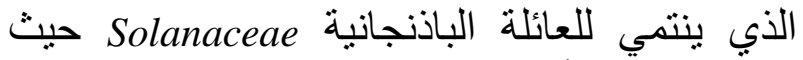
يحتوي في أجزائه الخضرية لفية لإنى السومنين و الويثانين Somnine حيوي ومواد أخرى دهنية ور اتتجية وزيوت طيارة Volatile oil كثير من الأحياء الدقيقة الضارة بالنبات بمن النبات.
مستخلص نبات الدفلة لله قرة ثنبيطية لنمو الفطر المسبب للعفن البني والأبيض على الأخشاب. وكذلك تتوافق النتائج مع ما وجده الدبعي الخلبدي،

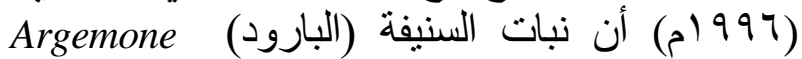
والذي ينتمي للعائلة الخشخاثية Papaveraceae

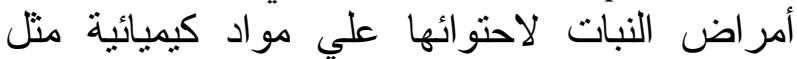

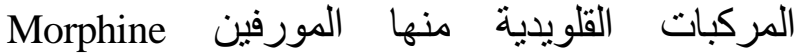

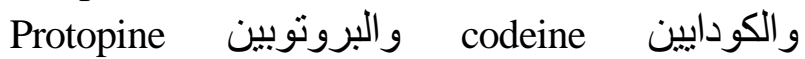

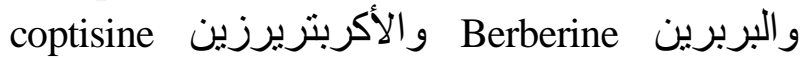

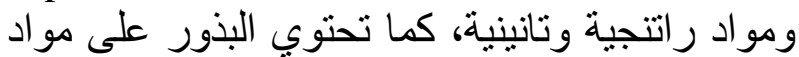

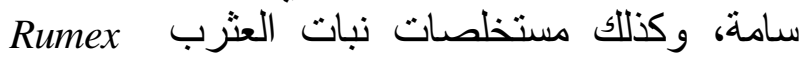

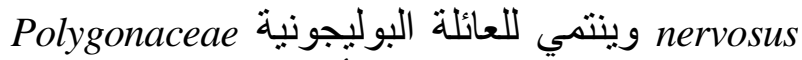
Oxalic acid يحتوي نبات العثرب على الأكسالات

جدول (ّ). تأثير المستخلصات النباتية على نسبة الإصابة بالعفن الفحمي ونمو نبات الفاصوليا بعد ه ؛ يوم من الزراعة (-post

\begin{tabular}{|c|c|c|c|c|c|c|}
\hline \multirow{2}{*}{\multicolumn{2}{|c|}{ الوزن الجاف للمجموع (جم) }} & \multirow{2}{*}{\multicolumn{2}{|c|}{ للمجموع (جم) الطري }} & \multirow{3}{*}{$\begin{array}{c}\text { [ للانخفاض في الإصابة } \\
\text { [\% reduction }\end{array}$} & \multirow{3}{*}{$\begin{array}{l}\text { (emer } \\
\text { \% لعدوث المرض } \\
\text { post - emergence } \\
\text { [\%] incidence }\end{array}$} & \multirow{3}{*}{$\begin{array}{l}\text { المعاملةة } \\
\text { Treatments }\end{array}$} \\
\hline & & & & & & \\
\hline الجذري & الخضري & الجذري & الخضري & & & \\
\hline $0.12 \mathrm{~B}$ & $2.99 \mathrm{~A}$ & $1.07 \mathrm{ABC}$ & $20.85 \mathrm{~A}$ & 47.82 & $40.00 \mathrm{~B}$ & السنيفة \\
\hline $0.15 \mathrm{AB}$ & $2.57 \mathrm{~A}$ & $1.36 \mathrm{AB}$ & $21.78 \mathrm{~A}$ & 84.77 & $11.67 \mathrm{C}$ & الكافور \\
\hline $0.12 \mathrm{~B}$ & $2.85 \mathrm{~A}$ & $0.89 \mathrm{BC}$ & $17.62 \mathrm{~A}$ & 39.12 & $46.67 \mathrm{~B}$ & العبب \\
\hline $0.21 \mathrm{~A}$ & $2.92 \mathrm{~A}$ & $1.58 \mathrm{~A}$ & $21.78 \mathrm{~A}$ & 43.48 & $43.33 \mathrm{~B}$ & العثرب \\
\hline $0.14 \mathrm{AB}$ & $2.24 \mathrm{~A}$ & $0.75 \mathrm{BC}$ & $16.51 \mathrm{~A}$ & 56.52 & 33.33 CB & الدفلة \\
\hline $0.12 \mathrm{~B}$ & $2.50 \mathrm{~A}$ & $0.99 \mathrm{ABC}$ & $19.20 \mathrm{~A}$ & 89.99 & $7.67 \mathrm{C}$ & الكنح \\
\hline $0.11 \mathrm{~B}$ & $2.09 \mathrm{~A}$ & $0.71 \mathrm{C}$ & $12.71 \mathrm{~A}$ & - & $76.67 \mathrm{~A}$ & الشـاهد \\
\hline 0.08 & NS & 0.63 & NS & & 28.06 & \%L.S.D at 5 \\
\hline
\end{tabular}

كما أوضحت النتائج أن متوسط الوزن الطري

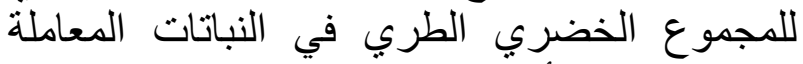

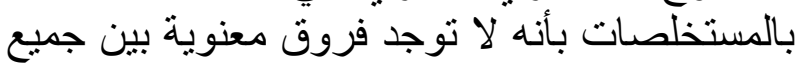

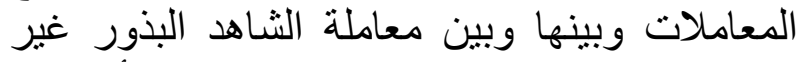

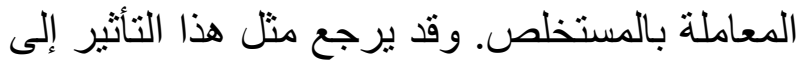

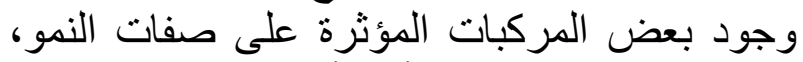
وقد لا يكون لمنل هذا التأثنير أهمية عند تهدية التدم النبات

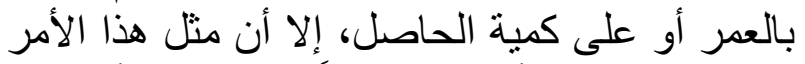

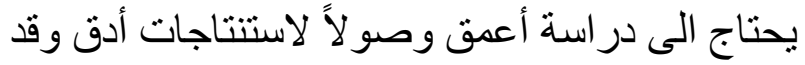

النسبة المئوية (\%) للانخفاض في حدوث المرض reduction

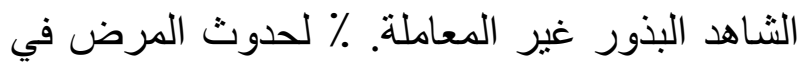

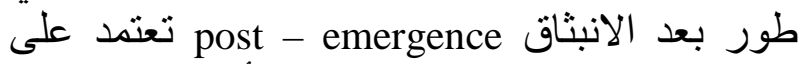
عدد النباتات التي تظهر علئ عليها الأعراض مقارنة بعدد النباتات الكلية النابتة. 
و العبب على الثاهد (بيئة غذائية بدون مستخلص)،

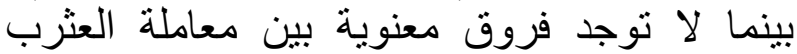

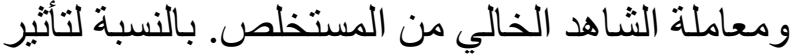
المستخلصات المائية النباتية على على نسبة الإنبات

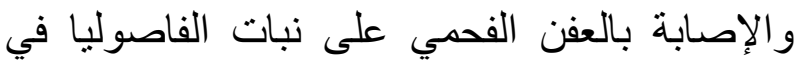

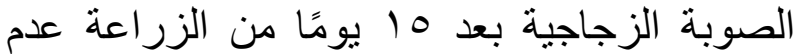

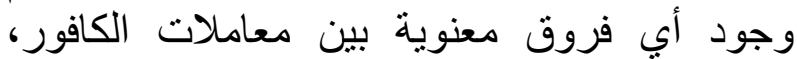

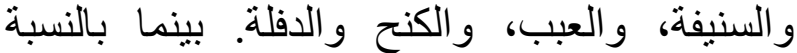

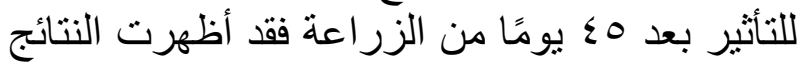
تفوق الكنح والكافور معنويًا على معاملات السنا من السنيفة،

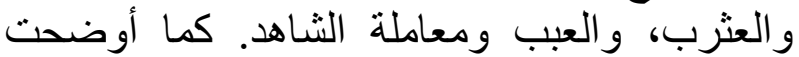

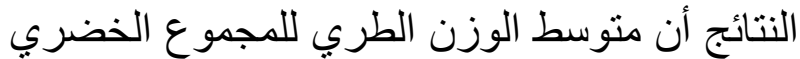

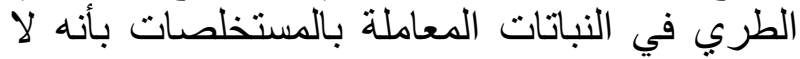

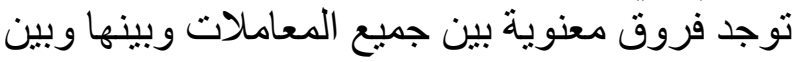
معاملة الثاهد. وبالنسبة للعثرب فئن فإن نتائج التحليل أظهرت تفوق معاملة العثرب معنويًا على معاملات

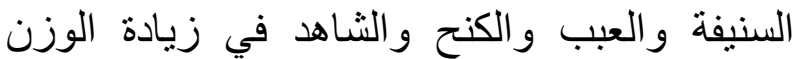
الجاف للجذور، بينما لم توجد فروق معنوية بين بقية

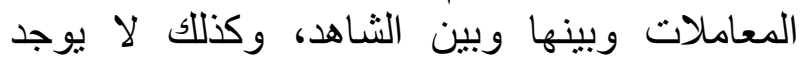
فروق معنوية بين العثرب و الكافور و الدفلة. و عليه

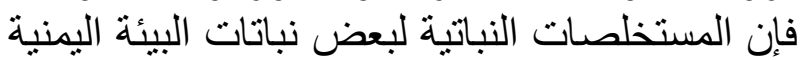

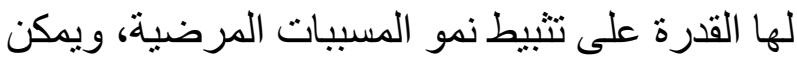

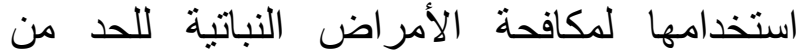

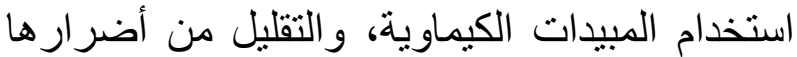

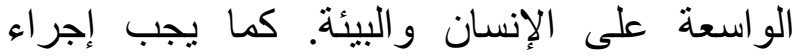
دراسات أوسع لمعرفة المو اد الفعالة في هذه النباتات الاتهات

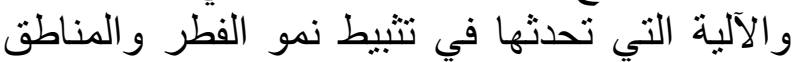
الحساسة من الفطر التي يحدث فيها تهو التأثير.

$$
\text { شكر وثقدير }
$$

نود أن نعرب عن شكرنا و عظيم امتنانتا لكل منتسبي

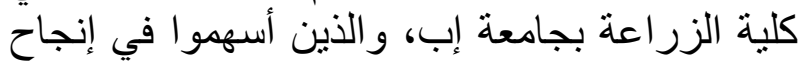

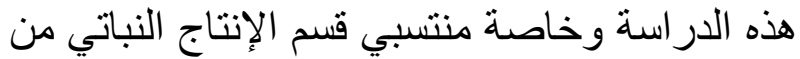

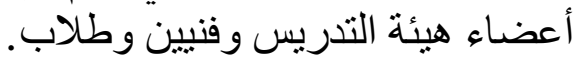

$$
\text { المراجــ }
$$

أولاً: المراجع العربية الجبوري، باقر عبد العزبة خلف والحيلر، حامد جعفر أبو بكر

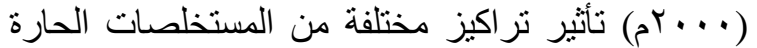
والباردة لبعض الأدغال في إنبات ونمو الحنطة Triticum
سبق الإشارة الى وجود مثل هذه التأثير ات من خلال

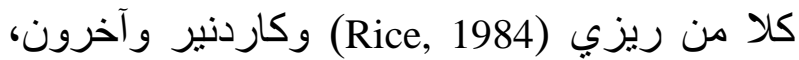
. (م) $99 \cdot$. (من

كما أظهرت النتائج في الجدول رقم (ب) عدم وجود أي فروق معنوية بين المعاملات وبين معاملة الثاهد

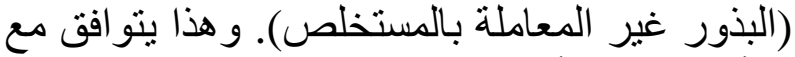

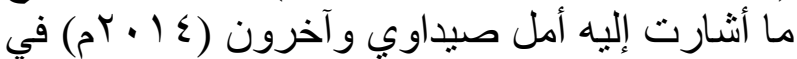
تأثثير التفاعل بين المستخلصات النهات النباتية وعزون لات فطر Macrophomina phaseolina (Tassi) Goid على طول جذور وسيقان بادرات السمسم، وتفوقت

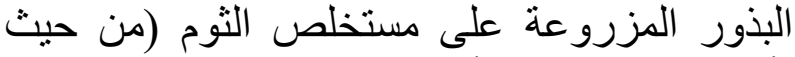

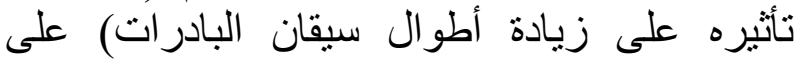
الثـاهد و عدم وجود فروق معنوية بادية بين المعاملات

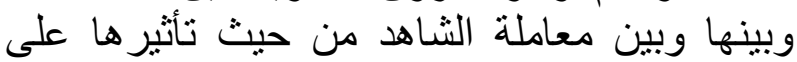
زيادة أطو ال جذور البادر ات. كما أوضحت نتائج التحليل تفوق معاملة العثرب

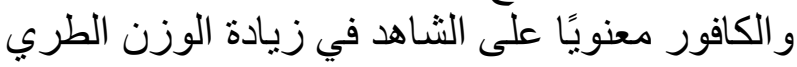

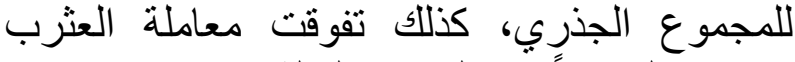

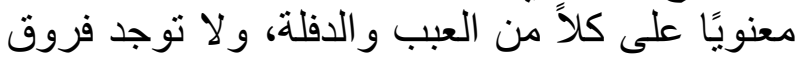

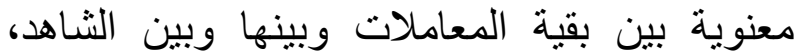
وأوضحت نتائج التحليل تفوق معاملة العثرب معنديًا على معاملات السنيفة والعبب و الكنح و الشاهد في زيادة الوزن الجاف للجذور ، ولا لا يوجد فروق معنوية

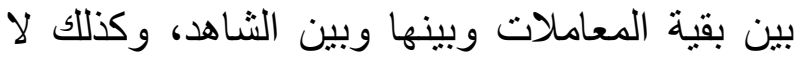
يوجد فروق معنوية بين العثرب و الكافور و الدفلة.

\section{؛. الملخص والاستنتاجات}

تمت هذه الدراسة في المختبر و الصوبة الزجاجية

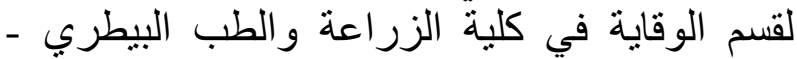

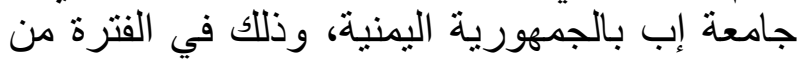

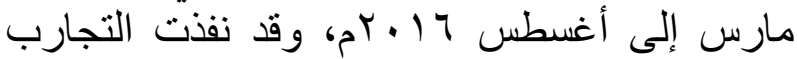
باستخدام التصميم العشوائي الكامل وحلات البيات البيانات إحصائيًا باستخدام جدول تحليل التباين، وتم تقدير

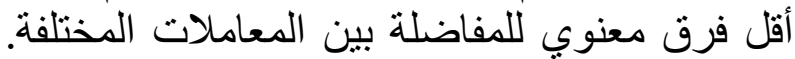

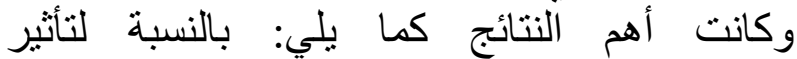

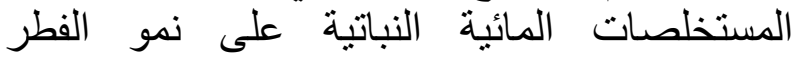
المدروس في المختبر فقد أظهرت النتائج تفوق كل من المعاملات الكنح، و الدفلة، و الكافور، و والسنيفة فوته 
Fusarium الكاتيوسان في بعض الخو اص الحيوية للفطر Oxysporum f. sp. Lycoperici

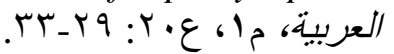

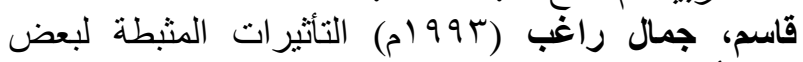

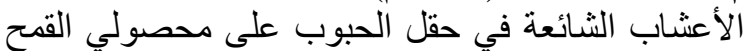

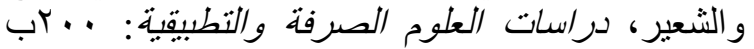

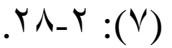

كاردنير، فرنكلين، واربرينت بيرس، وبات ووروجرال ميشيل

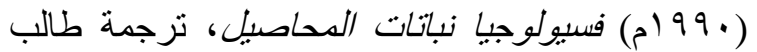

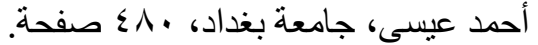

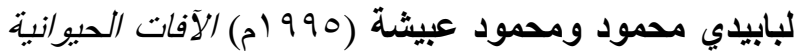

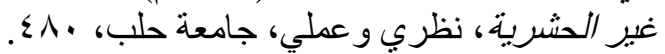

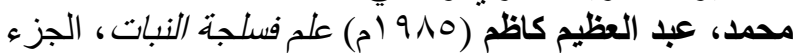

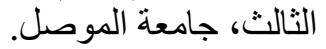

\section{ثانياً: المراجع الأجنبية}

Bhatt, B. P. and Todaria, N. P. (1990) Studies on allelopathic effect of some agro forestry tree crops of Garhwal Himalaya, Agro forestry systems, 12: 251-255.

Bost, S. (2006) Root Rot and seedling Disease of Bean and Peas. Plant Disease Extension, sp277-O. the Uni. of Tennessee.

EI-Fiki, A. I. I., Mohamed, F. G., EI- Deeb, A. A. and Kalifa, M. A. (2004) Some applicable methods for controlling sesame charcoal rot disease (Macrophomina phaseolina) under greenhouse conditions, Egyptian Journal of Phytopathology, 32 (1/2): 87-101.

El-Morsi, M.E.A. and Mahdy, H.A. (2013) Efficacy of some organic compounds against root rot wilt diseases of olive transplants and their growth response in new valley governorate, Egypt, Egypt. J. Agric. Res., 91 (2): 389-401.

El-Mougy, N.S., El-Gamal, N.G. and Abdel-Kader, M.M. (2007) Control of wilt and root rot incidence in Phaseolus vulgaris $\mathrm{L}$. by some volatile compounds, J. Plant Protection Res., 47 (3): 255-265.

Hadizadeh, I., Peivastegan, B. and Kolahi, M. (2009) Anti fungal activity of Nettle (Urtica dioica L.) Coloynth (Citrullus colocynthis L. Schrad) oleander (Nerium oleander $L$ ) and Konar (Zizuphus spina- christi L.) Extracts of plants pathogenic fungi, Pakistan Journal of Biological Science, 12 (1): 58- 63.

Haggage, W and EI-Sayed, M. (1998) New approaches for controlling soil borne fungi in infection cucumber plan under greenhouse condition university Ain Shams, The requirements for the degree of doctor of philosophy, 139.

Ionita, A., Lliescu, H., Jinga, V. and Iordache, E. (1995) Macrophomona Phaseolina dangerous parasite of cropped plants - possiboloties for control, Problem de-protectia Plantelor, 23 (2): 179 - 196. aestivum L. تأثير الأدغال الثتوية، مجلة جامعة بابل،

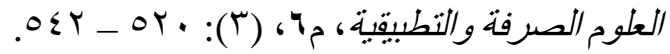

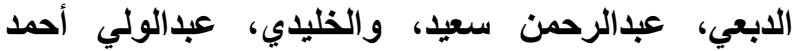

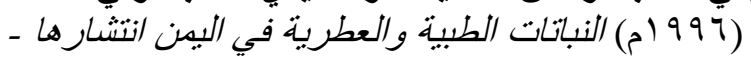

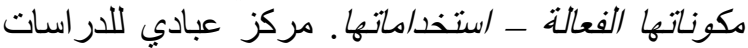

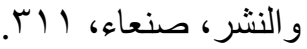
الراوي، خاشع محمود وخلف الله، عبدالعزيز ( (... بم)

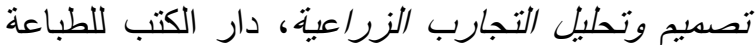

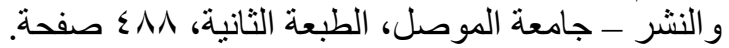

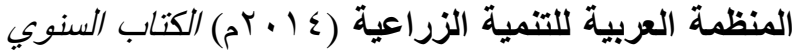

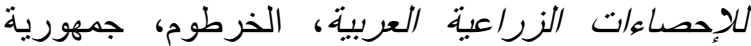

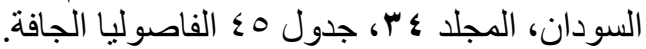

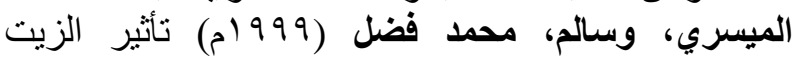
والمستخلص الإيثانولي لبذور النيم ( Azadirachta (indica A. Juss

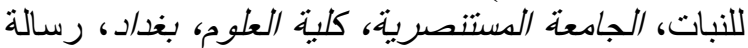

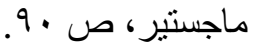

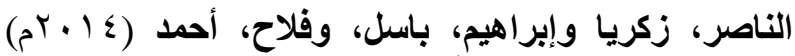

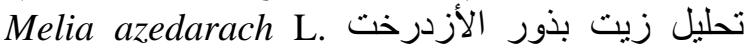

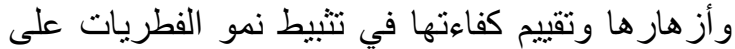

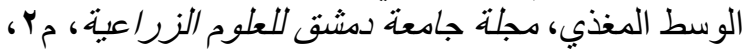

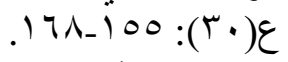

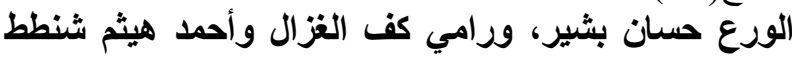

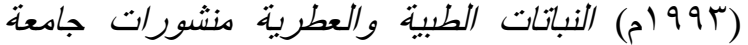

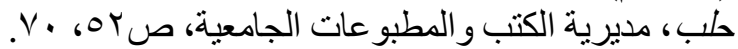

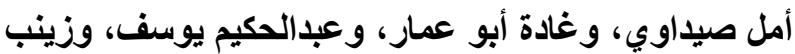

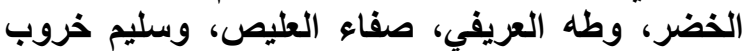

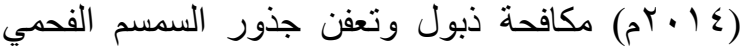
باستخدام بعض المستخلصات النباتية، المجلة الأردنية في الئي

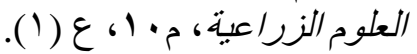

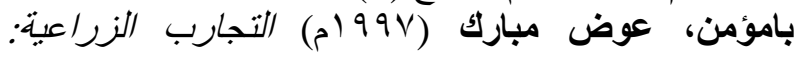

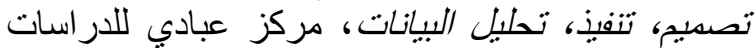

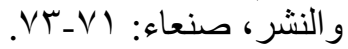

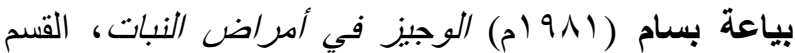

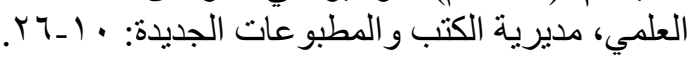

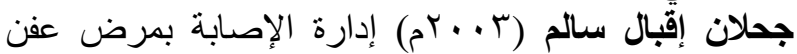

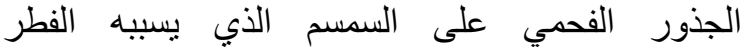
(Macrophomina phaseolina) الثمسي والتضاد الفطري وأساليب الزراعة اعنة، جامعة

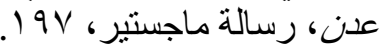

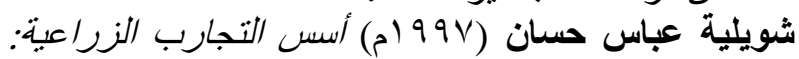
تصديم، تحليل، تطبيق، جامعة سبها، كلية الزر اعة: 77 التهاعل . $(1$

عبود مهدي، وأياد عبدالواحد الهيتي، فرقد عبدالرحيم

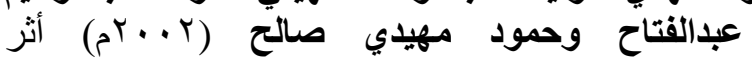


Riose, J.L., Recio, M. C. and Villar, A. (1987) Antimicrobial activity of selected plant employed in the Spanish Mediterranean area, J. Ethnoph armacol, 21: 139 - 152.

Siddiqui, I. A., Shaukat, S.S., Khan, G.H. and Zaki, M.J. (2002) Evaluation of Argemone Mexicana for Control of Root-Infecting Fungi in Tomato, Journal of Phytopathology, 150 (6): 321-329.

Sindhan, G. S., Hooda, I. and Parashar, R. D. (1999) Effect of some plant extracts on the vegetative growth of root rot causing fungi, Journal of Mycology and plant pathology, 29: $110-111$.

Smith, G. S. and Cravil, O. N. (1997) Field Screening of Commercial and Experimental Soybean Cultivars for their reaction to Macrophomina phaseolina, Plant Dis., 81 (4): 363-368.

Stampor-Chrzan, E. (2001) Antifungal activity of leaf and bark extracts on the growth and development of dampingoff fungi and their practical utilization in protection of seedling. Second European Allelopathy Symposium.

Unger, C. C. and Brideaux, G. B. (1997) Experiments from registration of biopesticiden in Sweden. EPPO Bulletin, 1 (27): 119-122.
Osman, G., Ramazan, M., Emin, D. M. O. and Melda, C.A. (2007) Application of extracts from poisonous plant, Nerium oleander $L$., as a wood preservation. African Journal of Biotechnology, 6 (17) :2000- 2003.

Papadopoulou, K., Melton, R.E., Leggett, M., Daniels, M.J. and Osboum, A.E. (1999) Compromised disease resistance in saponin-deficient. Plant Biol. 96(22):1292312928.

Paran, B.;Sharma, R. K., Singh, R. S ., Ghosh, A. C. and Baruah, P. (1996) Fungicidal activity of some Naturally occurring essential oils against Fusarium moniliforme, Journal of Essential oil Research, (8): 411-412 .

Phalisteen, S., Ishaq, S., Amardeep, K., Arif, J. and Sami, S. (2008) Evalution studies of some medicinal plant extracts and fungicides against Alternaria solani .African Journal of Clinical Experimental Mic ofrobiology, 9 (1) :19-25.

Rice, E. L. (1984) Allelopathy, Academic press, INC. New York, London.

Riebau, M., Berger, F. and Yegen, O. (1995) Chemical composition and fungitoxic properties to phytopathogenic fungi of essential oils of selected aromatic plants growing wild in Turkey, Journal of Turkey, 4 :399 -408.

\title{
Study the Effect of Water Extracts of Some Plants Against Fungus Macrophomina Phaseolina That Causes Charcoal Rot on Common Beans
}

\author{
*Ahmed A. Aljamali, Abdullah N. Al-Dhaibani Gamal A. Al-Shrabi and Marwan A. Manea \\ *Plant Production Department -Faculty of Agriculture - Ibb University, Republic of Yemen.
}


shoot. On the other hand, the results showed that the extract of Rumex nervosus and eucalyptus had positive results in fresh weight gain of the root system by 1.58, 1.36 g., respectively, comparing with 0.71 g. for the control. Rumex nervosus extract showed positive results as an increase in the dry weight of the roots, followed eucalyptus camaldulensis and Nerium oleander 0.21, 0.15, 0.14 g., respectively, comparing with the control $0.11 \mathrm{~g}$. Accordingly, the plant extracts of some plants from the Yemeni environment have the ability to inhibit the growth of pathogens and can be used to control plant diseases to reduce the use of chemical fungicides and reduce the extensive damage to humans and the environment.

Keywords: Plant extractions, fungus diseases, charcoal and beans. 